01,02

\title{
Влияние релаксации кубита на транспортные свойства микроволновых фотонов
}

\author{
(C) А.Н. Султанов, Я.С. Гринберг
}

Новосибирский государственный технический университет, Новосибирск, Россия

E-mail:: sultanov.aydar@ngs.ru

В работе с помощью метода неэрмитового гамильтониана исследовано прохождение одиночного фотона в одномерном волноводе, взаимодействующем с резонатором, содержащим произвольное число фотонов, и двухуровневый искусственный атом, с учетом релаксации последнего. Получены аналитические выражения для транспортных коэффициентов, в явном виде учитывающие параметр релаксации кубита. Форма коэффициента прохождения (отражения), когда в резонаторе находится более одного фотона, качественно отличается от однофотонного резонатора, и содержит в себе проявление эффекта фотонной блокады. Время жизни кубита зависит от числа фотонов в резонаторе.

Работа выполнена при финансовой поддержке Российского научного фонда (грант № 16-19-10069.)

DOI: 10.21883/FTT.2017.11.45041.08k

\section{1. Введение}

В настоящее время растет интерес к однофотонным процессам в твердотельных мезоскопических структурах, содержащих искусственные атомы - кубиты. В таких системах релаксация и декогеренция играют существенную роль в транспорте микроволновых фотонов. Как правило, теоретически транспортные коэффициенты с учетом затухания кубита рассчитываются с помощью введения оператора Линдблада в уравнение для матрицы плотности [1]. Таким способом в приближении слабого возбуждения можно получить аналитические выражения для коэффициента прохождения микроволнового фотона через открытый волновод, взаимодействующий с резонатором, в котором находится не более одного фотона $N=1$ [2]. Но уже для $N=2$ не удается найти аналитическое решение и соответствующую (формально бесконечную) цепочку уравнений Гейзенберга приходится решать численно [3]. В работе [4] с помощью метода неэрмитового гамильтониана были получены аналитические выражения для коэффициентов прохождения при произвольном числе фотонов в резонаторе, но без учета релаксации кубита. В настоящей работе релаксация кубита учитывается с помощью введения дополнительного канала распада в неэрмитовую часть гамильтониана. При этом связь системы с этим дополнительным каналом описывается как взаимодействие кубита с термостатом. При этом получаются аналитические выражения для коэффициентов прохождения, куда явным образом входит параметр релаксации кубита.

\section{2. Постановка задачи}

Исследуемая система описывается следующим полным гамильтонианом:

$$
H=H_{W}+H_{C}+H_{S}+H_{C-W}^{\mathrm{int}}+H_{C-S}^{\mathrm{int}}+H_{B-S}^{\mathrm{int}},
$$

где $H_{W}=\sum_{k} \hbar \omega_{k} c_{k}^{+} c_{k}$ описывает фотоны с частотой $\omega_{k}$ в волноводе. Волновод взаимодействует с фотонным резонатором (ФР), который предварительно содержит $N$ фотонов с фундаментальной частотой $\omega_{C}$ : $H_{C}=\hbar \omega_{C} a^{+} a$. В ФР расположен искусственный двухуровневый атом с энергией возбуждения $\hbar \Omega$, который может переходить в возбужденное состояние $|e\rangle$, поглощая фотон из резонатора, а также переходить в основное состояние $|g\rangle$, испуская фотон в резонатор или в термостат: $H_{s}=\hbar \Omega \sigma_{z} / 2$. Взаимодействие резонатора и волновода описывается гамильтонианом $H_{C-W}^{\mathrm{int}}=\hbar \xi \sum_{k}\left(c_{k}^{+} a+c_{k} a^{+}\right)$, где $\xi-$ параметр связи между резонатором и волноводом. Взаимодействие атома и резонатора описывается гамильтонианом Джейнеса-Каммингса $H_{C-S}^{\text {int }}=\hbar \lambda\left(a^{+} \sigma_{-}+a \sigma_{+}\right)$, где $\lambda$ - параметр взаимодействия между кубитом и резонатором. Взаимодействие с термостатом, описывающим процесс передачи энергии возбужденного атома в степени свободы, не связанные с наблюдаемыми фотонами, описывается гамильтонианом $H_{B-S}^{\text {int }}=\hbar \gamma \sum_{l}\left(b_{l}^{+}+b_{l}\right) \sigma_{x}$, где $\gamma$ - параметр взаимодействия кубита с термостатом, и последний описывается набором осцилляторов $H_{B}=\sum_{l} \hbar \omega_{l} b_{l}^{+} b_{l}$.

$a\left(^{\dagger}\right), c_{k}\left(c_{k}^{\dagger}\right), b_{l}\left(b_{l}^{\dagger}\right)$ представляют собой бозонные операторы уничтожения (рождения), а $\sigma_{x}, \sigma_{z}$ - спиновые операторы Паули, $\sigma_{-}=|g\rangle\left\langle e\left|, \sigma_{+}=\right| e\right\rangle\langle g|$. 
Для удобства введем следующие обозначения:

$$
\begin{gathered}
H_{e x}=H_{W}+H_{C-W}^{\mathrm{int}}+H_{B-S}^{\mathrm{int}}, \\
H_{\text {in }}=H_{C}+H_{S}+H_{C-S}^{\mathrm{int}} .
\end{gathered}
$$

Мы будем решать задачу в приближении однофотонных взаимодействий, что будет учтено выделением из Гильбертова пространства соответствующих состояний.

\section{3. Выбор состояний}

Состояние, означающее возбуждение осциллятора в термостате, обозначим как $\left|p_{\gamma}\right\rangle$. Тогда в рассматриваемой задаче по однофотонному транспорту можно ввести следующие векторы состояний:

$$
\begin{gathered}
\left|0_{w}\right\rangle \otimes\left|0_{\gamma}\right\rangle \otimes|g, N\rangle=|1\rangle, \\
\left|0_{w}\right\rangle \otimes\left|0_{\gamma}\right\rangle \otimes|e, N-1\rangle=|2\rangle,
\end{gathered}
$$

которые описывают ситуацию, когда в волноводе отсутствует фотон, и атом находится либо в основном, либо в возбужденном состоянии. Данные состояния условно можно принять за исходные, и поскольку исходное число фотонов $N$ выбирается произвольно, это ни на что в дальнейшем не повлияет. Теперь возможны следующие варианты эволюции этих векторов:

$$
\begin{aligned}
& \left|k_{w}\right\rangle \otimes\left|0_{\gamma}\right\rangle \otimes|g, N-1\rangle=|a, k\rangle, \\
& \left|k_{w}\right\rangle \otimes\left|0_{\gamma}\right\rangle \otimes|e, N-2\rangle=|b, k\rangle,
\end{aligned}
$$

когда из резонатора в волновод испустился один фотон; либо возбужденный атом отдаст энергию в термостат:

$$
\left|0_{w}\right\rangle \otimes|\gamma\rangle \otimes|g, N-1\rangle=|c, p\rangle \text {. }
$$

Мы не учитываем состояние $\left|k_{w}, p_{\gamma}, g, N-2\right\rangle$, поскольку амплитуда вероятности перехода в рамках однофотонных взаимодействий в него из состояний (4.1) равна нулю, что будет показано в разд. 5. В результате „непрерывного“ обмена фотонами резонатора с атомом возникают одетые состояния $|1, N\rangle,|2, N\rangle$ [суперпозиция состояний (4.1)] и $|1, N-1\rangle,|2, N-1\rangle$ [суперпозиция состояний (4.2)]. При этом уровень $|1, N\rangle$ лежит выше уровня $2, N\rangle$, и Раби - расщепление энергетических уровней между этими одетыми состояниями определяется уравнениями:

$$
\begin{gathered}
Q_{R}=\sqrt{\left(\omega_{c}-\Omega\right)^{2}+4 \lambda^{2} N}, \\
Q_{R}^{\prime}=\sqrt{\left(\omega_{c}-\Omega\right)^{2}+4 \lambda^{2}(N-1)},
\end{gathered}
$$

где (5.1) соответствует расщеплению между состояниями $|1, N\rangle,|2, N\rangle$, а $(5.2)$ - расщеплению между состояниями $|1, N-1\rangle,|2, N-1\rangle$.
Выражения (5) являются условием наблюдения фотонной блокады [5]. Данный эффект проявляется в том, что при малом числе фотонов, когда $\Omega_{R} \neq \Omega_{R}^{\prime}$, коэффициент прохождения резко изменяется при изменении числа фотонов в резонаторе на единицу.

Суперпозиционные состояния можно представить следующим образом:

$$
\begin{gathered}
|m, N\rangle=\alpha_{m}|1\rangle+\beta_{m}|2\rangle, \\
|j, N-1, k\rangle=a_{j}|a, k\rangle+\beta_{j}|b, k\rangle,
\end{gathered}
$$

где $j, m=1,2$, коэффициенты суперпозиции: $\alpha_{1}=\beta_{2}=$ $=\cos \theta, \quad \alpha_{2}=-\beta_{1}=\sin \theta, \operatorname{tg} 2 \theta=\frac{2 \lambda \sqrt{N}}{\omega_{C}-\Omega} ; \quad \alpha_{1}=\beta_{2}=\cos \theta^{\prime}$, $b_{2}=-a_{1}=\sin \theta^{\prime}, \operatorname{tg} 2 \theta^{\prime}=\frac{2 \lambda \sqrt{N-1}}{\omega_{C}}-\Omega$.

Суперпозиционным состояниям (6.1) соответствуют энергии $E_{m}=\omega_{c} N-\frac{1}{2} \omega_{c} \pm \frac{\Omega_{R}}{2}$, а состояниям (6.2) $E_{\Theta 1}=\omega_{c}(N-1)-\frac{1}{2} \omega_{c} \pm \frac{\Omega_{R}^{\prime}}{2}$.

\section{4. Проекционные операторы и эффективный неэрмитовый гамильтониан}

В рамках используемого метода неэрмитового гамильтониана выбранные состояния можно поделить на соответствующие дискретному (внутренние состояния) и непрерывному (внешние состояния) спектрам. Обозначим подпространство состояний с дискретным спектром как $Q(6.1)$, а подпространство состояний с непрерывным спектром как $P(6.2)$ и (4.3). Тогда проекционные операторы на данные подпространства можно представить как

$$
\begin{aligned}
p= & \sum_{k}|1, N-1, k\rangle\langle 1, N-1, k| \\
& +|2, N-1, k\rangle\left\langle 2, N-1, k\left|+\sum_{p}\right| c, p\right\rangle\langle c, p|
\end{aligned}
$$

и $q=|1, N\rangle\langle 1, N|+| 2, N\rangle\langle 2, N|$.

Переход между внешними состояниями описывается оператором $T[6]$

$$
\langle i|T| j\rangle \equiv T_{i j}=\sum_{\nu}\langle i|K| v\rangle\left\langle v\left|\left[1+\frac{j}{2} K\right]^{-1}\right| v\right\rangle,
$$

где индекс $v$ относится к внешним состояниям, а матричные элементы оператора $K$ вычисляются как

$$
\langle i|K| j\rangle \equiv K_{i, j}=\sum_{m} \frac{\bar{A}_{m}^{i} A_{m}^{j}}{E-E_{m}}
$$

где $i, j=1,2, c$, амплитуды $A_{m}^{j}$ характеризуют переход между подпространствами. Точные выражения для данных амплитуд можно записать, используя проекцию 


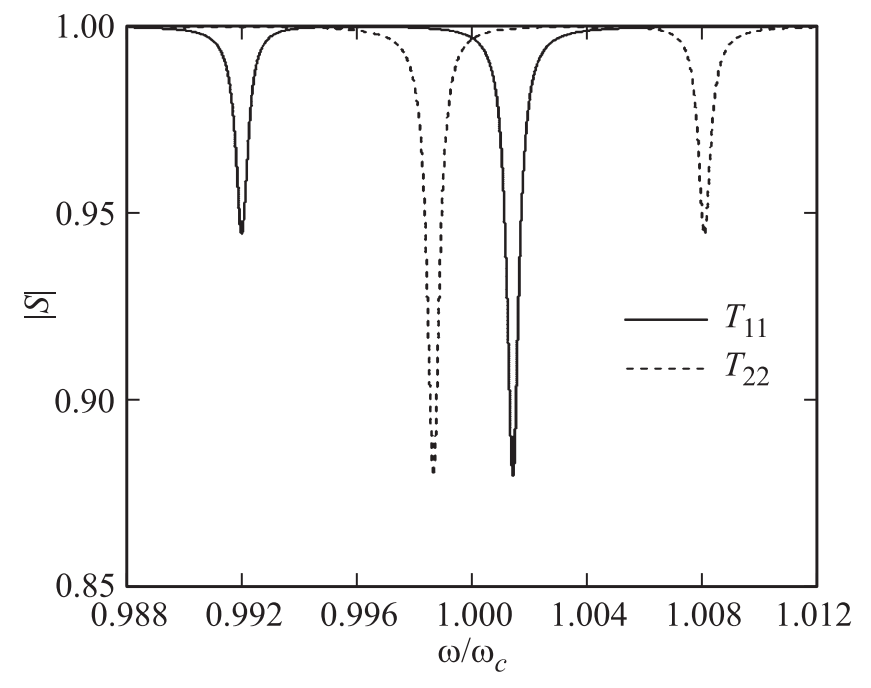

Рис. 1. Амплитудно-частотная характеристика в сильном резонансном режиме для потенциального рассеяния. Параметры системы: $\Omega=3 \mathrm{GHz}, \omega=3 \mathrm{GHz}, \Gamma=1 \mathrm{MHz}, \lambda=10 \mathrm{MHz}$, $\gamma_{2}=0.1 \Gamma, N=2$.

полного гамильтониана на ранее выбранные подпространства:

$$
\begin{gathered}
A_{m}^{1}=\left\langle m, N\left|H_{Q P}\right| 1, N-1, k\right\rangle, \\
A_{m}^{2}=\left\langle m, N\left|H_{Q P}\right| 2, N-1, k\right\rangle, \\
A_{m}^{c}=\left\langle m, N\left|H_{Q P}\right| c, p\right\rangle,
\end{gathered}
$$

где $H_{Q P}=Q H P$.

Коэффициент прохождения фотонов между внешними состояниями выражается через матрицу рассеяния $S$ с помощью известной формулы $S_{i, j}=\sigma_{i, j}-j T_{i, j}$. Рассчитывая таким образом элементы матрицы рассеяния $S$, можно получить аналитические выражения, описывающие транспорт единичного фотона в однокубитной структуре с $N$ фотонами в резонаторе и с учетом затухания кубита.

Приведем лишь конечные формулы и в следующем разделе проведем их краткий анализ.

$$
\begin{aligned}
& T_{11}=\langle 1 N-1|T| 1 N-1\rangle=\Gamma_{w} \frac{1}{4 \Omega_{R}^{\prime} D_{1}}\left[N\left(\Omega_{R}^{\prime}+\Delta\right)\right. \\
& \times\left(2 \delta_{t}+\Delta+\Omega_{R}^{\prime}\right)+(N-1)\left(\Omega_{R}^{\prime}-\Delta\right)\left(2 \delta_{t}-\Delta+\Omega_{R}^{\prime}\right) \\
& \left.+2 j \Gamma_{w} N(N-1) \Omega_{R}^{\prime}+8 \lambda^{2} N(N-1)+i \Gamma_{q} N Q_{R}^{\prime}+i \Delta \Gamma_{q} N\right],
\end{aligned}
$$

$$
T_{22}=\langle 2 N-1|T| 2 N-1\rangle=\Gamma_{w} \frac{1}{4 \Omega_{R}^{\prime} D_{2}}\left[N\left(\Omega_{R}^{\prime}-\Delta\right)\right.
$$$$
\times\left(2 \delta_{t}+\Delta-\Omega_{R}^{\prime}\right)+(N-1)\left(\Omega_{R}^{\prime}+\Delta\right)\left(2 \delta_{t}-\Delta-\Omega_{R}^{\prime}\right)
$$$$
\left.+2 j \Gamma_{w} N(N-1) \Omega_{R}^{\prime}-8 \lambda^{2} N(N-1)+i \Gamma_{q} Q_{R}^{\prime} N-i \Gamma_{q} \Delta N\right],
$$

$$
\begin{aligned}
T_{21} & =\langle 2 N-1|T| 1 N-1\rangle \\
& =-\Gamma_{w} \frac{\lambda \sqrt{N-1}}{2 \Omega_{R}^{\prime} D_{1}}\left[2 \delta_{t}-\Omega_{R}^{\prime}-\Delta+i N \Gamma_{q}\right], \\
T_{12} & =\langle 1 N-1|T| 2 N-1\rangle \\
& =-\Gamma_{w} \frac{\lambda \sqrt{N-1}}{2 \Omega_{R}^{\prime} D_{2}}\left[2 \delta_{t}+\Omega_{R}^{\prime}-\Delta+i N \Gamma_{q}\right],
\end{aligned}
$$

где $D_{i}$ содержат в себе комплексные резонансы системы:

$$
\begin{gathered}
D_{1}=\left(\omega-\omega_{1+}\right)\left(\omega-\omega_{1-}\right), \\
D_{2}=\left(\omega-\omega_{2+}\right)\left(\omega-\omega_{2-}\right), \\
\omega_{1 \pm}=\omega_{c}-\frac{\Omega_{R}^{\prime}}{2}-\frac{i}{4}\left[\Gamma_{w}(2 N-1)+\Gamma_{q}\right] \\
\pm \frac{1}{2} \sqrt{\left[\Delta-\frac{j}{2}\left(\Gamma_{w}-\Gamma_{q}\right)\right]^{2}+4 \lambda^{2} N,} \\
\omega_{2 \pm}=\omega_{c}+\frac{\Omega_{R}^{\prime}}{2}-\frac{i}{4}\left[\Gamma_{w}(2 N-1)+\Gamma_{q}\right] \\
\pm \frac{1}{2} \sqrt{\left[\Delta-\frac{j}{2}\left(\Gamma_{w}-\Gamma_{q}\right)\right]^{2}+4 \lambda^{2} N,}
\end{gathered}
$$

где введены расстройки $\Delta=\omega_{c}-\Omega$ и $\delta_{t}=\omega-\omega_{c}$, а также постоянные спонтанного излучения из резонатора в волновод $\Gamma_{w}=\frac{\xi^{2}}{v_{g}} L$ и релаксация кубита $\Gamma_{q} \approx \gamma^{2}$.

Формулы (10) учитывают влияние взаимодействий как на амплитуду, так и на фазу регистрируемого фотона на выходе системы. Так, при процессах, соответствующих уравнениям (10.1) и (10.2), внутренняя система и энергия налетающего фотона остается без изменений, что соответствует потенциальному рассеянию фотона. В случаях неупругого рассеяния (10.3) и (10.4) энергия фотонов изменяется на $\pm \hbar \Omega_{R}^{\prime}$, что также напрямую следует из закона сохранения энергии (для случая, когда мы пренебрегаем потерями). Резонансные частоты (12) соответствуют квадруплету, чисто квантовому проявлению эффекта, известного как триплет Моллоу. Основное отличие состоит в расщеплении центрального пика на два условно „центральных“, при этом стандартные „боковые“ пики триплета сохраняются. Типичный вид описанной амплитудно-частотной характеристики для процессов потенциального рассеяния, соответствующих уравнениям (10.1) и (10.2), представлен на рис. 1

Из уравнения (11) видно, что с ростом числа фотонов увеличивается ширина резонанса, и падает его амплитуда на определенной частоте, что экспериментально наблюдалось в работе [7]. На рис. 2 представлены АЧХ для разного числа фотонов в резонаторе вблизи боковых пиков, поскольку удобно смотреть именно здесь, так как они примерно пропорциональны $\omega_{c} \pm\left(\frac{\Omega_{R}^{\prime}}{2}+\frac{\Omega_{R}}{2}\right)$, а центральные пики $\omega_{c} \pm\left(\frac{\Omega_{R}^{\prime}}{2}-\frac{\Omega_{R}}{2}\right)$. 

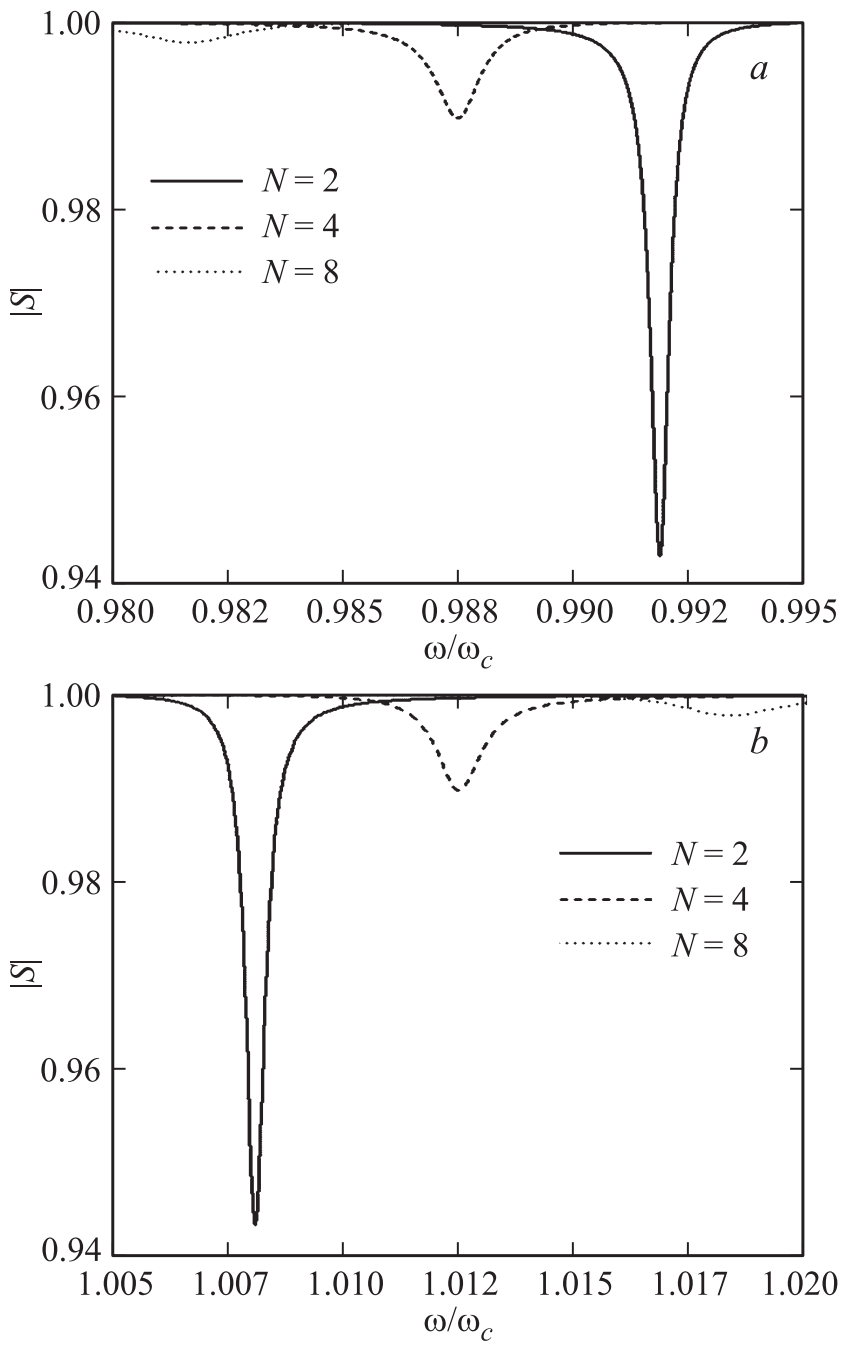

Рис. 2. Амплитудно-частотные характеристики коэффициента прохождения на боковых резонансах: $a-$ в сильном резонансном режиме $S 11 ; b-$ в сильном резонансном режиме $S 22$. Параметры системы те же, что и для рис. 1.

\section{5. Моделирование и анализ}

Рассмотрим достаточно простой случай, когда $N=1$. При этом все коэффициенты [кроме уравнения (10.1)] будут равны либо 1, либо 0. При этом выражение (10.1) упрощается

$$
\begin{aligned}
T_{11} & =\langle a|T| a\rangle \\
& =\Gamma_{w} \frac{\omega-\Omega+\frac{i}{2} \Gamma_{q}}{\left[\omega-\left(\Omega-\frac{i}{2} \Gamma_{q}\right)\right]\left[\omega-\left(\omega_{c}-\frac{j}{2} \Gamma_{w}\right)\right] \lambda^{2}} .
\end{aligned}
$$

Данное выражение совпадает с тем, что получается при решении уравнения эволюции с Линдбладианом [2]. В этом случае мы видим, что затухание вводится простым переходом к комплексным фундаментальным частотам. Эта формула была проверена многочисленными экспериментами [7-10]. В некоторых работах затухание при наличии многофотонного резонатора при фитингах описывалось как комплексная добавка к фундаментальной частоте кубита, тем не менее, как следует из формулы, при $N>1$ введение затухания не сводится к автоматической замене $\Omega \rightarrow \Omega-\frac{j}{2} \Gamma_{q}$.

На рис. 3 показаны зависимости амплитуды и фазы коэффициентов прохождения от параметра релаксации при различном числе фотонов в резонаторе вблизи фундаментальной частоты резонатора („центральные“ пики), на рис. 4 те же зависимости, только для фотона на боковых частотах. При этом параметры системы таковы, что $\omega_{c}=\Omega$. На фундаментальной частоте изменение числа фотонов на единицу приводит к значительным изменениям как фазы, так и амплитуды сигнала после потенциального рассеяния. При $N=1$ остается лишь одно состояние из уравнения (6.2), при котором в
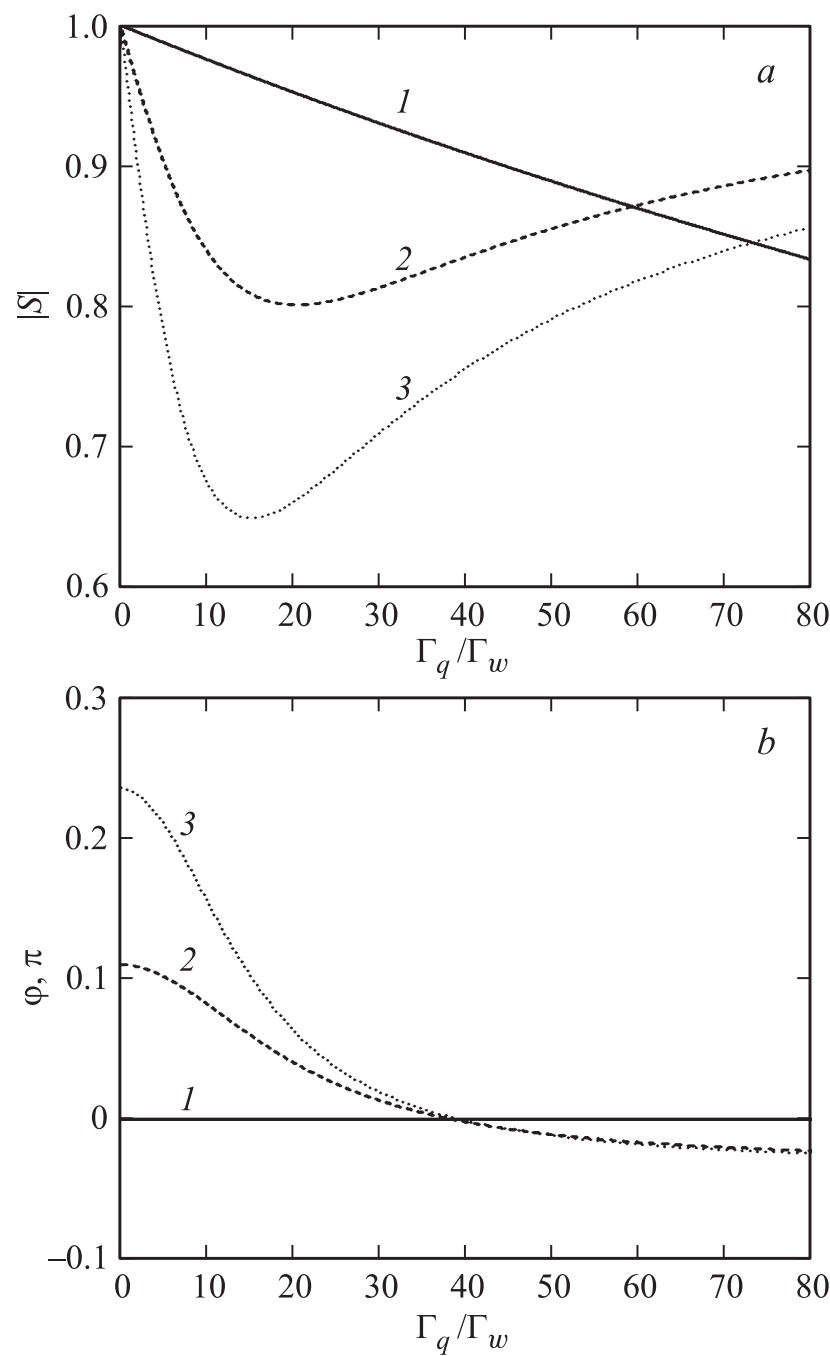

Рис. 3. Зависимость амплитуды $(a)$ и фазы $(b)$ коэффициента прохождения $S 11$ на фундаментальной частоте резонатора. Сплошная линия 1 соответствует формуле (46) из работы [2], пунктирные линии 2 и 3 соответствуют двум и трем фотонам. Параметры системы те же, что и для рис. 1. 

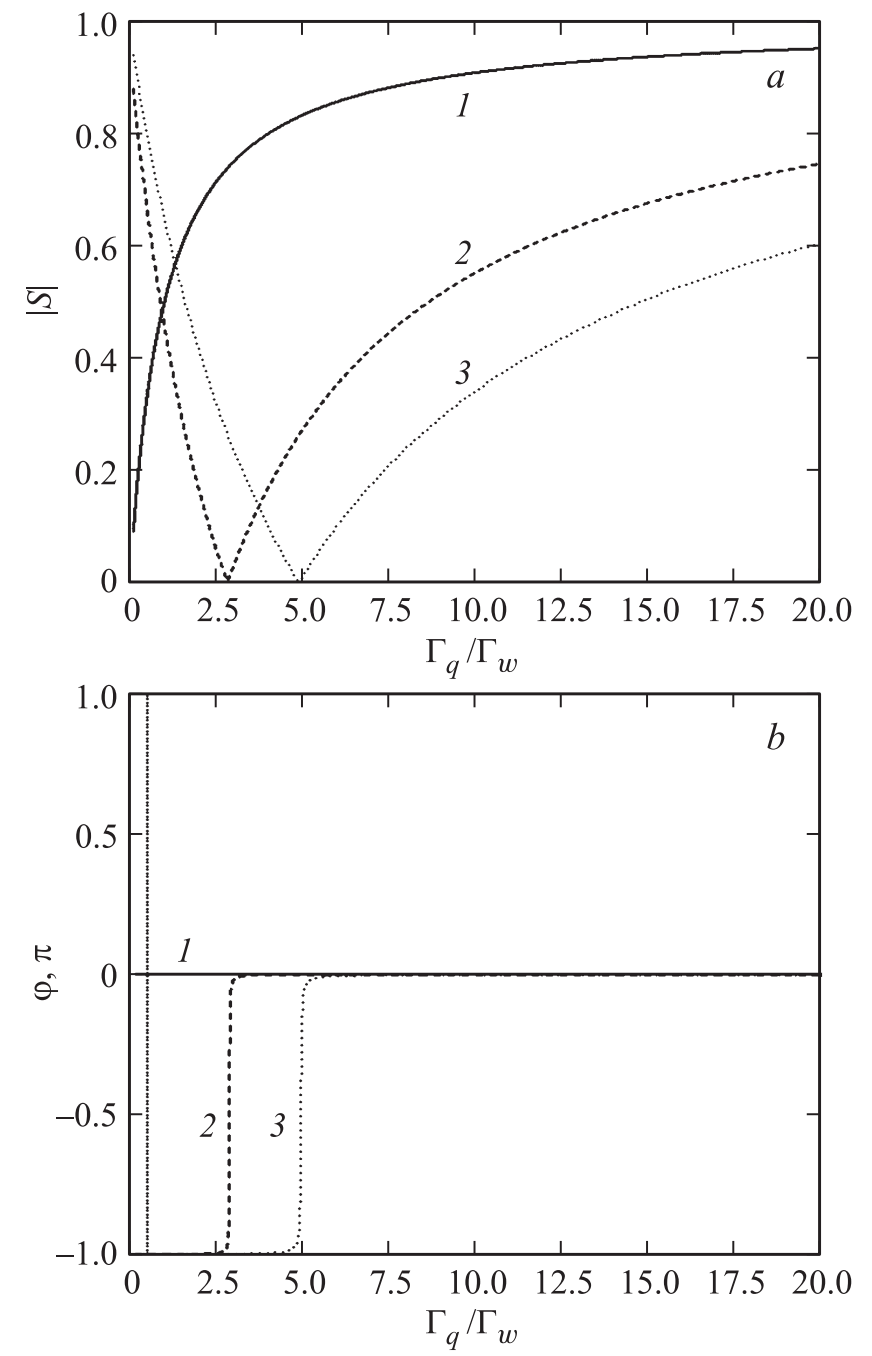

Рис. 4. Зависимость амплитуды $a$ и фазы $b$ коэффициента прохождения $S 11$ на центральных резонансах от релаксации кубита в сильном резонансном режиме. Сплошная линия 1 соответствует формуле (46) из работы [2], пунктирные линии 2 и 3 соответствуют двум и трем фотонам. Параметры системы: $\Omega=3 \mathrm{GHz}, \omega=3 \mathrm{GHz}, \Gamma=0.1 \mathrm{MHz}, \lambda=10 \mathrm{MHz}$.

резонаторе не остается фотонов ни до, ни после рассеяния, поэтому фаза сигнала не изменяется, поскольку формально это „тот же самый“ фотон. Параметр взаимодействия между фотонами и кубитом эффективно мал, поэтому амплитуда сигнала близка к единице при малых величинах релаксации кубита. С ростом релаксации амплитуда уменьшается по стандартному процессу диссипации энергии в термостат.

Добавление дополнительного фотона в резонатор предполагает, что взаимодействие фотонов с атомом происходит еще до рассеяния, и сам процесс рассеяния становится менее тривиальным. По виду фазовой зависимости на рис. $3, b$ можно предположить, что при малых значениях релаксации $\left(\Gamma_{q}: \Gamma_{w}\right)$ обратно в волновод вылетает фотон, успевший многократно провзаимодействовать с атомом. Этим же можно объяснить и быстрое уменьшение амплитуды сигнала, поскольку все фотоны через различные виртуальные переходы, автоматически учитывающиеся в данном формализме, теряют часть энергии. При дальнейшем увеличении постоянной релаксации атома амплитуда растет, и фаза коэффициента прохождения приближается к нулю, что говорит о том, что после рассеяния в волноводе наиболее вероятно зарегистрировать „тот же самый“ фотон. С другой стороны, точка минимума на амплитудной характеристике, как мы предполагаем, соответствует такому соотношению между параметрами релаксации и скорости испускания фотона из резонатора в волновод, когда фотоны в резонаторе (в нашей задаче их число „Поддерживается“ постоянным для каждого акта рассеяния) вероятнее отдают свою энергию в термостат, чем участвуют в волноводном транспорте, и с ростом релаксации эффективно приближается к ситуации, когда $N=1$, и релаксация мала (черная сплошная на рис. 3 ).

При регистрации фотонов на боковых частотах ситуацию уже не так легко описать, поскольку взаимодействие через виртуальные уровни в резонаторе носит весьма сложный характер. Из результатов моделирования можно сделать вывод лишь о том, что при двух фотонах не происходит их взаимодействия с атомом, и поэтому наиболее вероятно зарегистрировать „тот же самый далека от $\Omega$, это следует из того, что фаза практически не меняется с ростом релаксации. Тем не менее при малых значениях релаксации существенна разница в амплитуде проходящего сигнала для разного числа фотонов в резонаторе. Поскольку амплитуда $S 11$ может быть интерпретирована как вероятность обнаружить фотон в волноводе после потенциального рассеяния, то с увеличением числа фотонов в резонаторе и при их малой диссипации в термостат растет и вероятность вылета фотона в волновод, что интуитивно кажется логичным. С ростом релаксации различие между ситуациями с разным числом фотонов уменьшается, что, как предполагалось ранее, свидетельствует об эффективном наличии только одного фотона в резонаторе. Более того, с ростом релаксации вероятность обнаружить фотон на этой частоте растет, что также означает уменьшение вероятности перехода между одетыми состояниями из $P$ и $Q$ подпространств, поскольку в этом случае Рабиколебания затухают быстрее (время жизни одетых состояний уменьшается), чем фотон успевает высветиться в волновод. На рис. 5 мы видим, что с ростом релаксации амплитуда приближается к 1 даже для $N=1$. Это означает „расползание“ центрального провала, что формально означает, что при значительно больших постоянных релаксации вероятность фотона провзаимодействовать с системой приближается к 0 , и соответственно вероятность зарегистрировать его в волноводе - к 1 (это также видно по фазовой характеристике). При $N=2,3$ эта вероятность падает до какого-то значения релаксации $\Gamma_{q}^{2 p}$, а затем начинает также приближаться к 1. $\Gamma_{q}^{2 p}$ определяется как числом фотонов в резонаторе, 

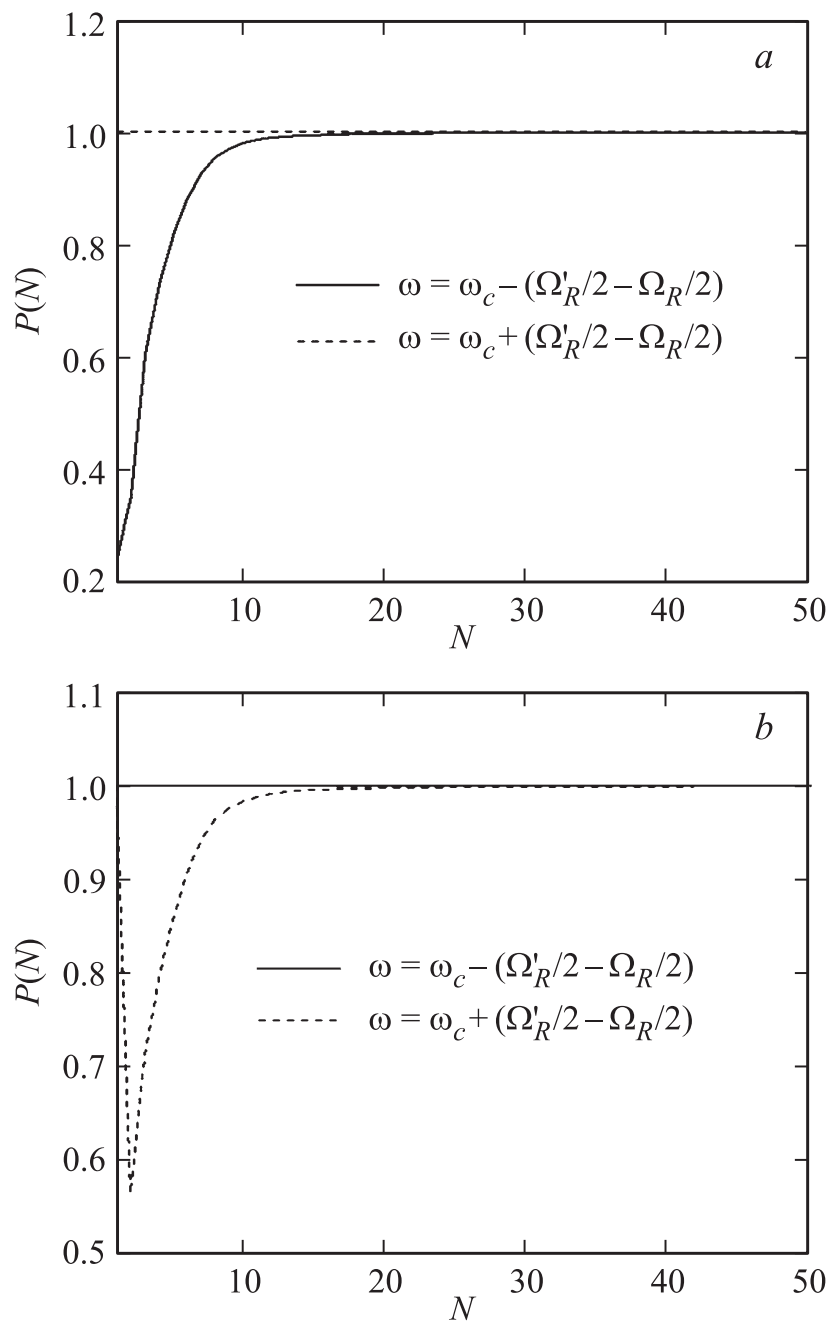

Рис. 5. Фотонная блокада на различных резонансных частотах при исходном состоянии $|N-1\rangle(a),|2 N-1\rangle(b)$.

так и постоянной испускания фотонов в волновод. Эту величину можно интерпретировать следующим образом: при $\Gamma_{q}<\Gamma_{q}^{2 p}$ Раби-колебания между одетыми состояниями затухают медленно, так что вероятность фотона попасть в систему (определяемая $\Gamma_{w}$ и наличием одетых уровней) отлична от нуля. После попадания фотона в систему есть вероятность фотона рассеяться в термостат, при этом падает вероятность обнаружить фотон в волноводе. При $\Gamma_{q}>\Gamma_{q}^{2 p}$ одетые состояния пропадают раньше, чем фотон успевает провзаимодействовать с системой (при $\Gamma_{w}<\Gamma_{q}$ ), и пропадают все уровни, на которые может попасть фотон, кроме $|a\rangle$, и поэтому зависимость приближается к случаю $N=1$. Этот эффект схож с эффектом блокады.

Одетые состояния полностью пропадают, когда все фотоны в резонаторе рассеиваются в термостат, а поскольку скорость рассеивания в термостат определяется величиной $\Gamma_{q}$, то с ростом числа фотонов граничное значение релаксации сдвигается в сторону бо́льших величин, и аналогичный эффект происходит с увеличе- нием $\Gamma_{w}$. При этом независимо от величины $\Gamma_{w}$ относительное положение граничных релаксаций сохраняется и для приведенных чисел фотонов в резонаторе будет $\Gamma_{q}^{N=2} / \Gamma_{w} \approx 2.83, \Gamma_{q}^{N=3} / \Gamma_{w} \approx 4.9$.

Фотонная блокада проявляется при малом числе фотонов в резонаторе, и поскольку, как было сказано ранее, эффект проявляется в резком изменении коэффициента прохождения фотона на определенной частоте при изменении числа фотонов на единицу, можно ввести функцию, описывающую степень этого изменения:

$$
P=\frac{|T[\omega(N), N]|}{|[w(N), N+1]|},
$$

где $\omega(N)=\omega_{c} \pm\left(\frac{\Omega_{R}^{\prime}}{2}-\frac{\Omega_{R}}{2}\right)-$ частота, соответствующая провалам на АЧХ. То есть при проявлении фотонной блокады $P$ будет сильно отличаться от единицы.

На рис. 5 показано, что в диапазоне малого числа фотонов, где $\Omega_{R} \neq \Omega_{R}^{\prime}$, явно проявляется фотонная блокада, которая при увеличении числа фотонов исчезает, т. е. $P=1$. В зависимости от частоты налетающего фотона блокада будет происходить при различных исходных состояниях.

\section{6. Заключение}

Нами получены аналитические выражения в чисто квантовом режиме, описывающие вероятности процессов потенциального и не потенциального рассеивания одиночного фотона на однокубитной системе с резонатором, в котором имеются $N$ фотонов. Для $N>1$ параметр релаксации кубита входит нетривиально, поскольку учитывает взаимодействие системы с термостатом во всех порядках, так, в отличие от уравнений эволюции, не приходится искусственно обрывать цепочку уравнений для операторов. Полученные формулы в квазистационарном приближении описывают изменение вероятности обнаружения фотона при различных соотношениях между постоянными релаксации и испускания фотонов в волновод, и могут быть использованы для описания эффекта фотонной блокады. Полученные выражения (10) могут быть использованы для случая, когда $\frac{\omega_{c}-\Omega}{\omega_{c}}<1$, так как мы учитывали только однофотонное взаимодействие.

\section{Список литературы}

[1] М.О. Скалли, М.С. Зубайри. Квантовая оптика. Пер. с англ. М.: ФИЗМАТЛИТ, 2003.

[2] А.Н. Омельчук, С.Н. Шевченко, Я.С. Гринберг, О. Астафьев, Е. Ильичев. Физика низких температур. 36, 1117 (2010).

[3] R. Bianchetti, S. Filipp, M. Baur, J.M. Fink, M. Goppl, P.J. Leek, L. Steffen, A. Blais, A. Wallraff. Phys. Rev. A 8, 043840 (2009).

[4] Ya.S. Greenberg, A.N. Sultanov. Phys. Rev. A 95, 053840 (2017). 
[5] A.J. Hoffman, S.J. Srinivasan, S. Schmidt, L. Spietz, J. Aumentado, H.E. Tureci, A.A. Houck Phys. Rev. Lett. 107, 053602 (2011).

[6] N. Auerbach, V. Zelevinsky. Rep. Progr. Phys. 74, 106301 (2011).

[7] A.F. van Loo, A. Fedorov, K. Lalumière, B.C. Sanders, A. Blais, A. Wallraff. Science 342, 1494 (2013).

[8] И.Л. Новиков, Б.И. Иванов, А.Н. Султанов, Я.С. Гринберг, Е.В. Ильичев. ФТТ 58, 2085 (2016).

[9] G. Oelsner, S.H.W. van der Ploeg, P. Macha, U. Hübner, D. Born, S. Anders, E. Il'ichev, H.-G. Meyer, M. Grajcar, S. Wünsch M. Siegel, A.N. Omelyanchouk, O. Astafiev. Phys. Rev. B 81, 172505 (2010).

[10] S.N. Shevchenko, G. Oelsner, Ya.S. Greenberg, P. Macha, D.S. Karpov, M. Grajcar, U. Hubner, A.N. Omelyanchouk, E. Il'ichev. Phys. Rev. B 89, 184504 (2014). 